(2) Open Access Full Text Article

ORIGINAL RESEARCH

\title{
Assessment Of Circulating Endothelial Cells And Their Progenitors As Potential Biomarkers Of Disease Activity And Damage Accrual In Behçet's Syndrome
}

This article was published in the following Dove Press journal:

Open Access Rheumatology: Research and Reviews

\section{Alberto Floris (D) \\ Matteo Piga (D) \\ Silvia Pinna \\ Maria Maddalena Angioni (D) \\ Mattia Congia (D) \\ Piero Mascia (D) \\ Elisabetta Chessa \\ Ignazio Cangemi (D) \\ Alessandro Mathieu \\ Alberto Cauli}

Rheumatology Unit, AOU University Clinic and University of Cagliari, Cagliari, Italy
Correspondence: Matteo Piga Rheumatology Unit, University Clinic AOU of Cagliari, SS 554, Monserrato, CA 09042, Italy

Tel +390706754069

Fax +390705 I3157

Email matteopiga@unica.it
Purpose: To explore the potential role of circulating endothelial cells (CECs) and their progenitors (EPCs) as biomarkers of disease activity and damage accrual in patients with Behçet's syndrome (BS), by using a standardised and reliable flow cytometry protocol.

Patients and methods: CECs and EPCs were assessed in 32 BS patients and 11 gender/age/ smoking habits matched healthy controls (HC). They were identified by flow cytometry as alive/ nucleated/CD45-negative/CD34-bright/CD146-positive and alive/nucleated/CD45-negative/ CD34-bright/CD309-positive events, respectively. In BS patients, demographic and clinical features, including disease activity (assessed by Behçet's disease current disease activity form, BDCAF) and irreversible damage accrual (by the vasculitis damage index, VDI) were recorded. Uni- and multivariate analysis were performed to compare the CECs and EPCs concentrations in BS vs HC and to identify potential associations with demographic or clinical features.

Results: The CECs concentration was significantly higher in the BS patients than HCs [median (IQR) $15.0(7.5-23.0)$ vs 6.0 (2.0-13.0) $\mathrm{CECs} / \mathrm{mL}, \mathrm{p}=0.024]$. In BS patients, no significant associations were found between CECs and demographic features, present and past clinical manifestations, BDCAF score and ongoing treatment. A significant association was observed between CECs and organ damage, as assessed by the VDI (rho 0.356, $\mathrm{p}=0.045$ ). Higher levels of CECs were especially associated with vascular damage [median (IQR) $23.0(14.0-47.0)$ vs $13.0(6.0-19.0) \mathrm{CECs} / \mathrm{mL}, \mathrm{p}=0.011]$, including arterial aneurysm and stenosis, complicated venous thrombosis, cerebrovascular accident. The concentration of EPCs did not significantly differ between the BS and HC [median 26.5 (13.0-46.0) vs 19.0 (4.0-42.0) EPCs $/ \mathrm{mL}, \mathrm{p}=0.316]$ and no significant associations were observed between their levels and any clinical characteristic.

Conclusion: Our study suggests that the CECs concentration is significantly higher in BS than healthy subjects, and it mainly correlates with vascular damage. A longitudinal extension of the present study on a wider cohort would be useful to validate the potential role of CECs as a marker or, hopefully, predictor of vascular damage in BS.

Keywords: circulating endothelial cells, CECs, endothelial progenitor cells, EPCs, Behçet's syndrome

\section{Introduction}

Behçet's syndrome (BS) is a multisystem relapsing inflammatory disorder, characterized by oral and genital aphtosis, skin lesions, uveitis, and potential vascular, neurologic and gastrointestinal involvement. ${ }^{1}$ The etiology of BS remains 
unknown, although the most widely held pathogenic hypothesis is that of aberrant autoinflammatory response triggered by an environmental agent in genetically susceptible subjects. ${ }^{2,3}$ Supporting this is the geoepidemiology of the disease and the association with polymorphisms in the HLA complex, particularly the HLA-B*51. ${ }^{4-6}$

Histopathologic studies demonstrated that the predominant lesion in BS is vasculitis, affecting both the vessel wall and perivascular tissues. Leukocytoclastic vasculitis, fibrinoid necrosis of postcapillary venules, or perivascular neutrophilic accumulations are some of the reported findings in the early stages of the cutaneous lesions. ${ }^{7}$ Similarly, neutrophil infiltrate and endothelial cell activation are recorded in the vasa vasorum of patients with BS and major vessels involvement. ${ }^{8}$

Circulating endothelial cells (CECs) and their progenitors (EPC) are restricted subpopulations of peripheral blood cells involved in the endothelial homeostasis. ${ }^{9}$ CECs are characterized by mature endothelial features and detach from vessel walls following vascular damage or the physiological tissue turnover. ${ }^{10} \mathrm{EPCs}$, characterized by an immature phenotype, are bone marrow resident cells, mobilized upon specific stimulation. Once in the bloodstream, EPCs home to target tissues where they are involved in endothelial repair or remodeling. ${ }^{9}$

Abnormalities in CECs and EPCs concentrations have been recorded in several vasculitides. ${ }^{11}$ Woywodt et al found that the concentrations of CECs were higher in patients with ANCA-associated vasculitis (AAV), when compared with healthy controls (HC) or patients with infections and other non-ANCA associated glomerulonephritis. Further, a significant association between CECs levels and disease activity was recorded in these patients (rho 0.704, $\mathrm{p}>0.0001$ ). ${ }^{12}$ Similarly, in Kawasaki disease, the mean number of CECs was found to be significantly higher in patients with vasculitis than in $\mathrm{HC}$, especially in the acute and subacute phases. ${ }^{13}$ In regard to EPCs, Závada et al found that patients with AAV have a significant and persistent deficiency of circulating EPCs when compared with HC, assuming an impaired mechanism of vascular repair that may contribute to repeated relapses in these patients. ${ }^{14}$ On the other hand, Nakatani et al found higher levels of EPCs following an increase in CECs in Kawasaki disease, particularly in patients with complicated coronary artery lesions, suggesting that EPCs may be involved in the repair of endothelial damage. $^{13}$
Poor and contrasting data on abnormalities in CECs and EPCs, derived from inadequate methodologies, are currently available in BS. ${ }^{15,16}$

This study aimed to evaluate whether the concentrations of CECs and EPCs, as assessed by a standardized flow cytometry protocol, are increased in patients affected by BS and significantly correlate with clinical features. It would provide explorative data on the vascular involvement in the disease pathogenesis and the potential role of these cells as biomarkers of disease activity or damage accrual.

\section{Methods}

\section{Patients And Controls}

In this cross-sectional cohort study, 32 unselected consecutive adult patients diagnosed with $\mathrm{BS}$ according to the International Criteria for Behçet's Disease (ICBD) ${ }^{17}$ were recruited at the Rheumatology Unit of the University Clinic of Cagliari. Further, 11 gender, age, and smoking habits matched HC were investigated. Subjects with infective, neoplastic or not BS-related cardiovascular disease were excluded.

The study was approved by the Local Ethical Committee (N. 2018/6028) and written informed consent was obtained from all subjects. All procedures were in accordance with the Good Clinical Practice standards and Helsinki Declaration.

\section{Clinical Assessment}

Demographic and clinical data, as well as ongoing treatment, were recorded for each patient at recruitment. Present and past clinical manifestations were categorized according to the ICBD criteria (oral aphtosis, genital ulcers, pathergy positivity, ocular, vascular central nervous system involvement), including also arthritis and gastrointestinal involvement. Erythrocyte sedimentation rate and C-reactive protein (CRP) values were collected as laboratory parameters. Disease activity was measured by using the Behçet's Disease Current Activity Form (BDCAF), both as continuous (total BDCAF score) and binominal variable (active: $\mathrm{BDCAF} \geq 1$, inactive $\mathrm{BDACF}=0$ ). ${ }^{18}$ The Physician Global Assessment (PGA) of disease activity on a 0 to 10 visual analogic scale was also recorded. Finally, according to the OMERACT (Outcome Measures in Rheumatology) recommendations, irreversible organ damage was also assessed in our study. However, as no specific damage assessment tools are currently available for BS, the vasculitis damage index (VDI) was used as a surrogate, being the most employed in other systemic 
vasculitides and being already applied in other cohorts including BS. ${ }^{19-21}$ Thus damage, defined as irreversible scars of previous disease activity or its treatment lasted for at least 3 months, was recorded both as a continuous variable, reflecting the overall extension of damage accrual (total VDI score) and binary variable, reflecting the presence or absence of any irreversible damage (VDI $\geq 1$ and VDI $=0$, respectively).${ }^{19}$ For the purpose of the study, items included in the cardiovascular and peripheral-vascular sections of the VDI, including also cerebrovascular were classified as vascular damage.

\section{Collection Of Blood Specimen}

Peripheral blood was drawn from the antecubital vein with $21 \mathrm{G}$ needles in EDTA vacutainer tubes. The first harvested $3 \mathrm{ml}$ tube was used to determine sample leukocyte count, in order to assess dual-plaform counting. It was excluded from the direct analysis of CECs and EPCs, to avoid counting cells derived from vascular damage caused by venipuncture.

\section{CECs And EPCs Count}

CECs determinations were performed within $4 \mathrm{hrs}$ from the sample collection by a standardized flow cytometry procedure, recently described elsewhere. ${ }^{10}$ Briefly, blood volume containing $20 \times 10^{6}$ leukocytes underwent erythrocyte-lysis with $40 \mathrm{~mL}$ of Pharm Lyse solution (BD Biosciences), followed by centrifugation and wash with $2 \mathrm{~mL}$ of Stain Buffer containing bovine serum albumin (BD Biosciences). Surface staining was accomplished by adding the resuspended pellet of each sample to the CEC Lyotube kit (Becton Dickinson, Custom), consisting in a panel tube (CD146PE, CD34PE-Cy7, CD309AlexaFluor647, CD45APC-H7, 7AAD) and a control tube (IsotypePE, CD34PE-Cy7, IsotypeAlexaFluor647, CD45APC-H7, 7AAD), and $1 \mu \mathrm{M}$ Syto-16 (Thermo Fisher Scientific, Eisai, Medipost - US) was added as liquid drop-in. ${ }^{10}$ Samples incubated in the dark for 30 mins at $4{ }^{\circ} \mathrm{C}$ were then washed and re-suspended in 1.5 $\mathrm{mL}$ of FACSFlow (BD Biosciences). CECs were defined as alive, nucleated, CD45-negative, CD34-bright, CD146-positive events (Figure 1); whereas, EPCs as alive nucleated, CD45-negative, CD34-bright and CD309-positive cells. ${ }^{9,10}$ a

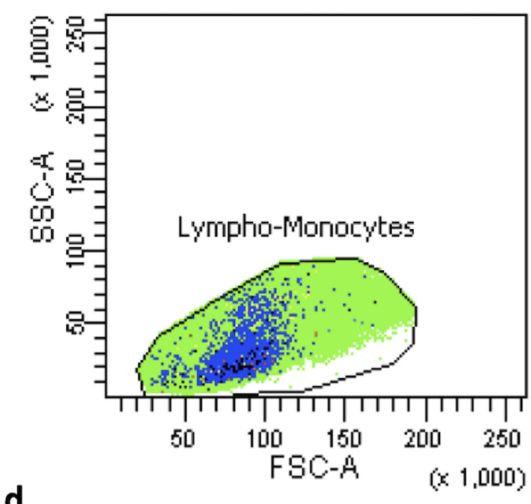

d

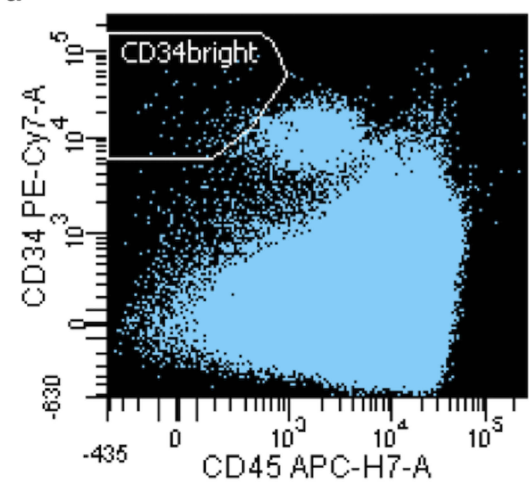

b

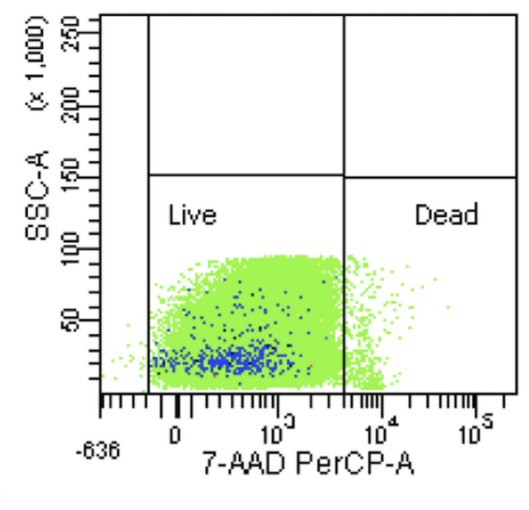

e

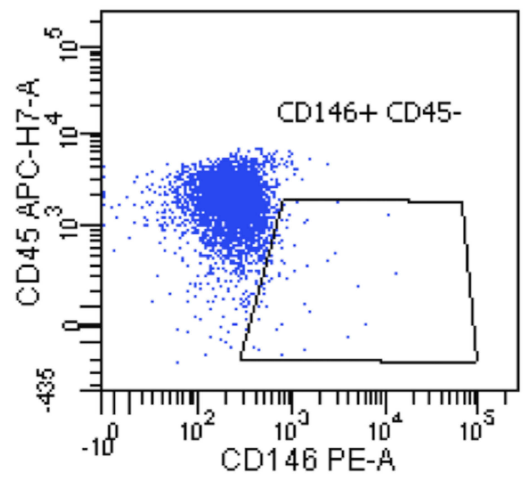

C

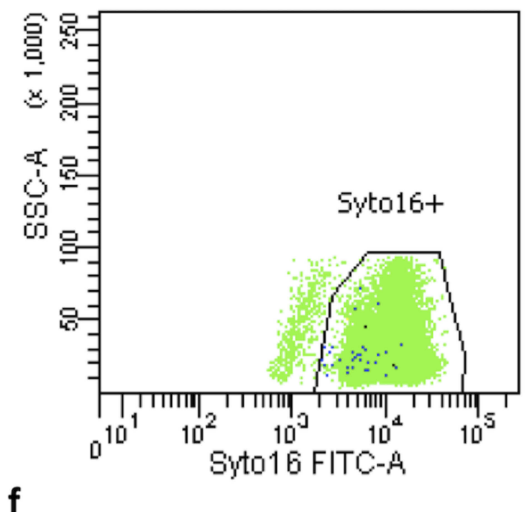

f

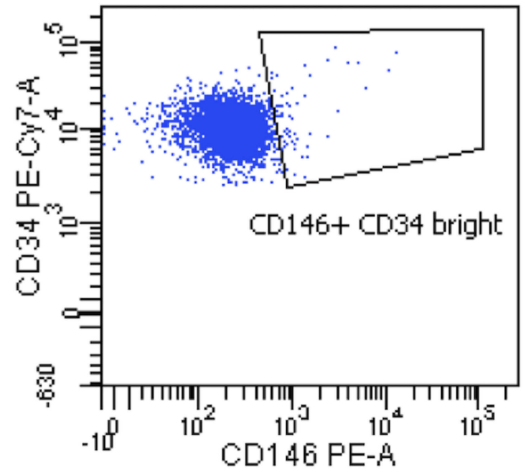

Figure I Flow cytometry identification of circulating endothelial cells (CECs). (A) Lympho-monocytes were gated in a forward scatter versus side scatter (FSC-A/SSC-A) dot plot. (B) Dead cells were excluded (7-AAD/SSC-A dot plot) and (C) nucleated events (Sytol6pos) were selected (Sytol6/SSC-A dot plot). (D) Events characterized by lympho-monocytes features, alive, nucleated, expressing bright levels of CD34 and CD45neg were identified. Analysis for CDI46 surface expression both on a CDI46/ CD45 (E) and CDI46/CD34 (F) dot plot was performed. The CEC compartment (CD34bright/CD45neg/CDI46pos cells) was identified. Gates were drawn based on the signal of the control tube, containing the corresponding isotype control in combination with all the remaining surface reagents. 
Finally, $2.5 \times 10^{6}$ events/sample with lympho-monocyte morphology were acquired by flow cytometry (FACSCanto II, BD Biosciences). A threshold combination was set on FSC and FITC channel (Syto16) to exclude very small and non-nucleated events. Data were analyzed using FACSDiva v 8.0.1 (BD) software.

\section{Statistical Analysis}

Standard descriptive statistics were used to summarize the demographic and clinical features of the studied cohort. Continuous data were expressed as mean $\pm \mathrm{SD}$ and/or median (IQR). $t$-Student test and Mann-Whitney $U$-test were used, when appropriate, to assess the association of CECs and EPCs with dichotomic clinical variables. Spearman's rank correlation coefficient was calculated to estimate the association between CECs and EPCs and continuous clinical variables. Linear multiple regression was used to further assess the independent association of baseline clinical features and levels of CECs and EPCs. P-values $<0.05$ were regarded as statistically significant. All analyses were performed using SPSS version 24.

\section{Results}

\section{Patients And Controls}

Details on demographic and clinical data from the study cohort are reported in Table 1. As expected, no significant differences were recorded between BS and HC groups, in terms of gender distribution (males $53.1 \%$ and $45.5 \%$, respectively, $\mathrm{p}=0.929$ ), age at recruitment (mean 49.6 \pm 11.3 and $44.8 \pm 12.6, \mathrm{p}=0.239$ ), and smoking habits (19.6\% and $18.2 \%, p=1.000)$.

\section{CECs}

The number of CECs was significantly higher in BS patients [median (IQR) 15.0 (7.5-23.0), range 0-48 $\mathrm{CECs} / \mathrm{mL}$ ] than HC [median (IQR) 6.0 (2.0-13.0), range $0-18] \mathrm{CECs} / \mathrm{mL}(\mathrm{p}=0.024)$ (Figure 2). The association of CECs levels with BS was confirmed after correction for sex, age, and smoking habits $(\mathrm{p}=0.040)$.

Within the BS group, no significant associations were found between CECs and demographic features, past or present clinical manifestations, BDCAF, PGA, and ongoing treatment $(\mathrm{p}>0.05)$ (Table 2$)$. CECs levels significantly correlated with the VDI, both in terms of total score (Spearman's coefficient $0.356, \mathrm{p}=0.045$ ) and occurrence of any damage [median (IQR) 19.0 (13.0-27.0) vs
Table I Baseline Features

\begin{tabular}{|c|c|c|c|}
\hline & BS $(n=32)$ & $H C(n=I I)$ & $\mathbf{p}$ \\
\hline \multicolumn{4}{|l|}{ Demographics } \\
\hline Males & $17(53.1 \%)$ & $5(45.5 \%)$ & 0.929 \\
\hline Mediterranean Europe origin & $30(93.8 \%)$ & II (100\%) & 1.00 \\
\hline Assessment age & $49.6(11.3)$ & $44.8(12.6)$ & 0.239 \\
\hline Onset age & $33.5(10.5)$ & - & \\
\hline Diagnosis age & $37.5(11.3)$ & - & \\
\hline Disease duration & $12.2(10.6)$ & - & \\
\hline HLA-B5I & $7 / 19(36.8 \%)$ & - & \\
\hline Current smoker & $6(19.4 \%)$ & $2(18.2 \%)$ & 1.00 \\
\hline \multicolumn{4}{|l|}{ Ever occurred manifestations } \\
\hline Oral aphtosis & $32(100 \%)$ & - & \\
\hline Genital aphtosis & $22(68.8 \%)$ & - & \\
\hline Skin lesions & $26(81.3 \%)$ & - & \\
\hline Ocular involvement & $17(53.1 \%)$ & - & \\
\hline CNS lesions & $3(9.4 \%)$ & - & \\
\hline Vascular lesions & 7 (21.9\%) & - & \\
\hline Pathergy test & $4(12.9 \%)$ & - & \\
\hline Arthritis & II (34.4\%) & - & \\
\hline GI involvement & I (3.I\%) & - & \\
\hline \multicolumn{4}{|l|}{ Active manifestations } \\
\hline Oral aphtosis & 4 (12.5\%) & & \\
\hline Genital aphtosis & $0(0 \%)$ & & \\
\hline Skin lesions & 5 (15.6\%) & & \\
\hline Ocular involvement & $2(6.3 \%)$ & & \\
\hline CNS lesions & $0(0 \%)$ & & \\
\hline Vascular lesions & I (3.1\%) & & \\
\hline Pathergy test & $0(0 \%)$ & & \\
\hline Arthritis & $0(0 \%)$ & & \\
\hline GI involvement & I (3.I\%) & & \\
\hline ESR & $19.0(19.5)$ & & \\
\hline CRP & $4.0(4.5)$ & & \\
\hline \multicolumn{4}{|l|}{ Clinimetric indices } \\
\hline BDCAF score & $2.3(2.8)$ & - & \\
\hline $\mathrm{BDCAF} \geq 1$ & $16(50.0 \%)$ & - & \\
\hline VDI score & $1.0(1.6)$ & - & \\
\hline VDI $\geq 1$ & $17(53.1 \%)$ & - & \\
\hline PGA & $2.75(2.4 I)$ & - & \\
\hline \multicolumn{4}{|l|}{ Treatment } \\
\hline Colchicine & $8(25 \%)$ & - & \\
\hline Glucocorticoids & $22(68.8 \%)$ & - & \\
\hline Conventional immunosuppressant & $15(46.8 \%)$ & & \\
\hline Azathioprine & $13(40.6 \%)$ & & \\
\hline Methotrexate & I (3.1\%) & & \\
\hline Thalidomide & I (3.1\%) & & \\
\hline Biologic immunosuppressant & $6(18.6 \%)$ & - & \\
\hline TNF inhibitor & $5(15.6 \%)$ & & \\
\hline ILIR inhibitor & I (3.1\%) & & \\
\hline
\end{tabular}

Notes: Categorical variables are expressed as number (\%); continuous variables as mean (SD).

Abbreviations: BS, Behçet's syndrome; $\mathrm{HC}$, healthy controls; CNS, central nervous system; Gl, gastrointestinal; BDCAF, Behçet's Disease Current Activity Form; VDI, vasculitis damage index; PGA, Physician Global Assessment; TNF, tumour necrosis factor; ILIR, interleukin I receptor. 


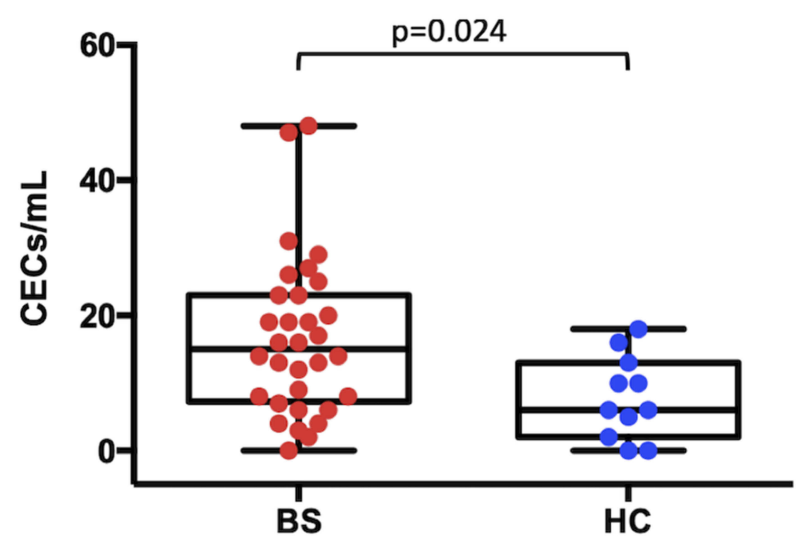

Figure 2 Concentration of circulating endothelial cells (CECs) in Behçet's syndrome (BS) patients and healthy controls $(\mathrm{HC})$. The ends of the box are the Ist and $3 r d$ interquartile, the vertical line inside the box is the median, the whiskers represent the highest and lowest values.

$12.0(6.0-16.0) \mathrm{CECs} / \mathrm{mL}$ in $\mathrm{VDI} \geq 1$ and $\mathrm{VDI}=0$, respectively; $\mathrm{p}=0.027$ ] (Figure 3).

Higher levels of CECs were especially associated with vascular damage [median (IQR) $23.0(14.0-47.0)$ vs 13.0 (6.0-19.0) $\mathrm{CECs} / \mathrm{mL}, \mathrm{p}=0.011]$, including arterial aneurysm, arterial stenosis, complicated venous thrombosis, cerebrovascular accident.

\section{EPCs}

The concentration of EPCs did not significantly differ between the BS [median (IQR) 26.5 (13.0-46.0), range 0-310 EPCs $/ \mathrm{mL}$ ] and HC groups [median (IQR) 19.0 (4.0-42.0), range 0-162 EPCs $/ \mathrm{mL}, \mathrm{p}=0.316$ ] (Figure 4).

Within the BS group, the highest value of EPCs (310 $\mathrm{EPCs} / \mathrm{mL}$ ) was recorded in a patient who had ongoing deep venous thrombosis on one lag. On univariate analysis, EPCs concentrations significantly correlated with recruitment age (rho 0.361; $\mathrm{p}=0.042$ ), disease duration (0.377, $\mathrm{P}=0.036$ ), and CRP (0.408; $\mathrm{p}=0.03$ ) (Table 2). However, these associations were not confirmed in multivariate analysis.

\section{Discussion}

BS is a complex multisystem disease, where the wide heterogeneity in clinical presentation and the lack of reliable biomarkers often result in significant difficulties in terms of diagnosis, monitoring and prognostic assessment. On the basis of the experience gained in other vasculitides, this study aimed to provide reliable data on the potential role of CECs and EPCs as biomarkers of disease activity or irreversible damage accrual in BS.
In our cohort, we found a significantly increased number of CECs in BS patients when compared with HC. However, no significant associations with current disease activity or other specific clinical patterns were recorded, with the exception for vascular irreversible damage. These findings suggest that, in the subset of patients with vascular involvement, the endothelial dysfunction with detachment of CECs persists even during the inactive periods. On the other hand, the lack of significant association with disease activity may be due to that all recruited patients were already on treatment, a few of them were on high disease activity and only a subject had a vascular active manifestation.

In a previous study, Kulty et al found that BS patients, especially in the active period, have elevated numbers of CECs compared with healthy controls. Conversely, in other studies, where BS was included among other vasculitides, no increase in CECs concentration was demonstrated. ${ }^{16}$

Compared with previous studies, our findings on CECs in $\mathrm{BS}$ are the first derived by a validated flow cytometry protocol. Indeed, most data on CECs are so far obtained by immunomagnetic separation protocols, where endothelial cells are isolated from whole blood with magnetic particles coated with anti-endothelial antibodies (eg, anti-CD146) and counted by fluorescence microscopy. ${ }^{11}$ However, this methodology is affected by significant limits in terms of reliability caused by the inter-operator variability and the potential aspecific binding of leukocytes to beads. ${ }^{11}$ In this context, a significant advance for the reliable identification of CECs was recently made with the validated flow cytometry protocol proposed by Lanuti et al in $2018^{10}$ and applied in our study, where CECs are defined as alive, nucleated, CD45-negative, CD34-bright, CD146-positive events.

In regard to the EPCs, in our BS cohort they were not significantly increased, and no significant associations were observed between their levels and any clinical characteristic. Further, no significant association was recorded between EPCs and CECs concentration. Similarly, Bozkirli et $\mathrm{al}^{22}$ observed that EPCs levels were comparable in patients with BS and $\mathrm{HC}(\mathrm{p}=0.849)$ and they did not correlate with disease activity or other clinical features, with the exception of thrombosis. Thus, our and previous data suggest that no abnormalities in repair system of endothelial damage are present in BS.

However, also for EPCs some methodological considerations are needed. Indeed, significant issues exist on the best cell surface markers to distinguish EPCs from mature 


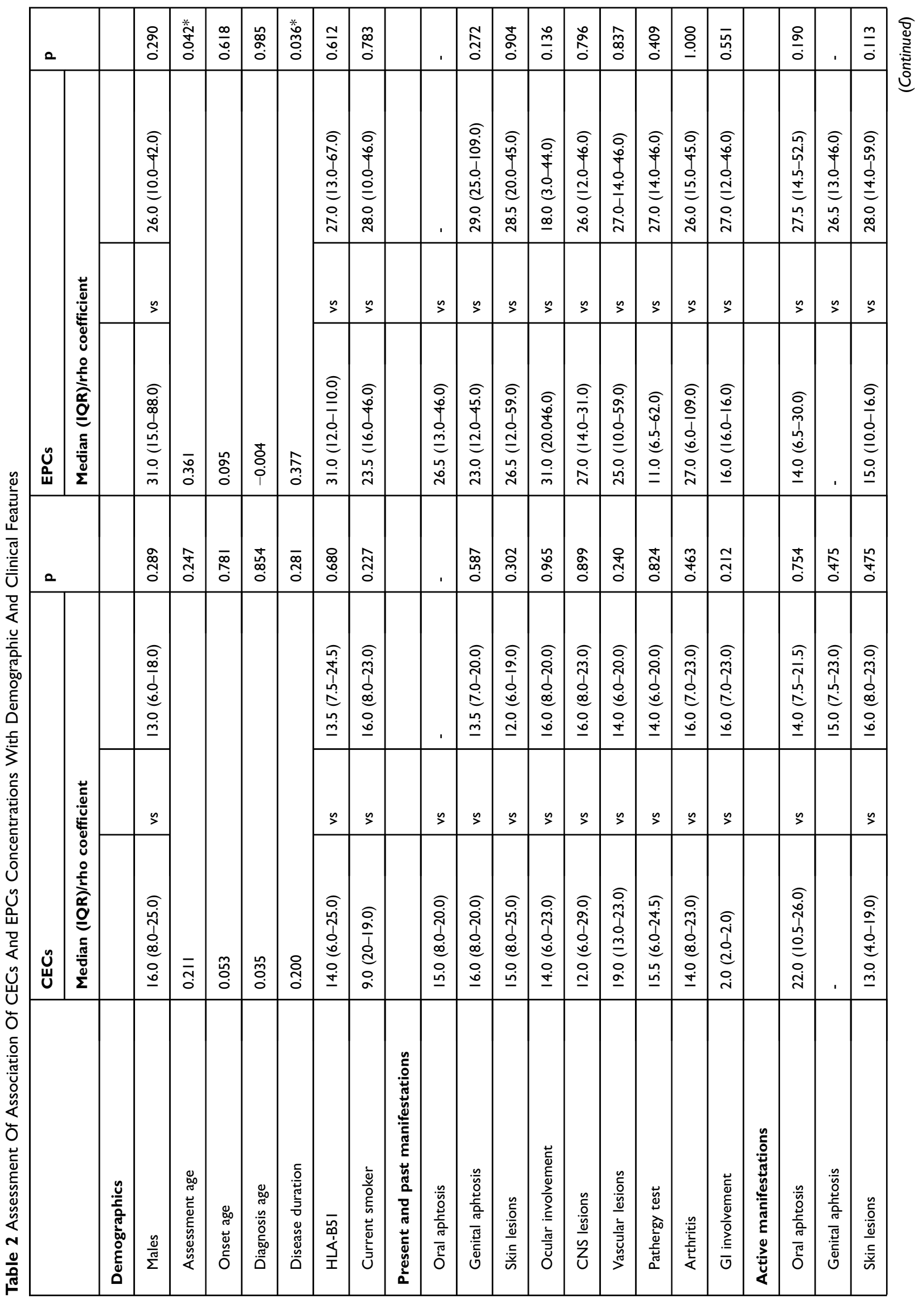




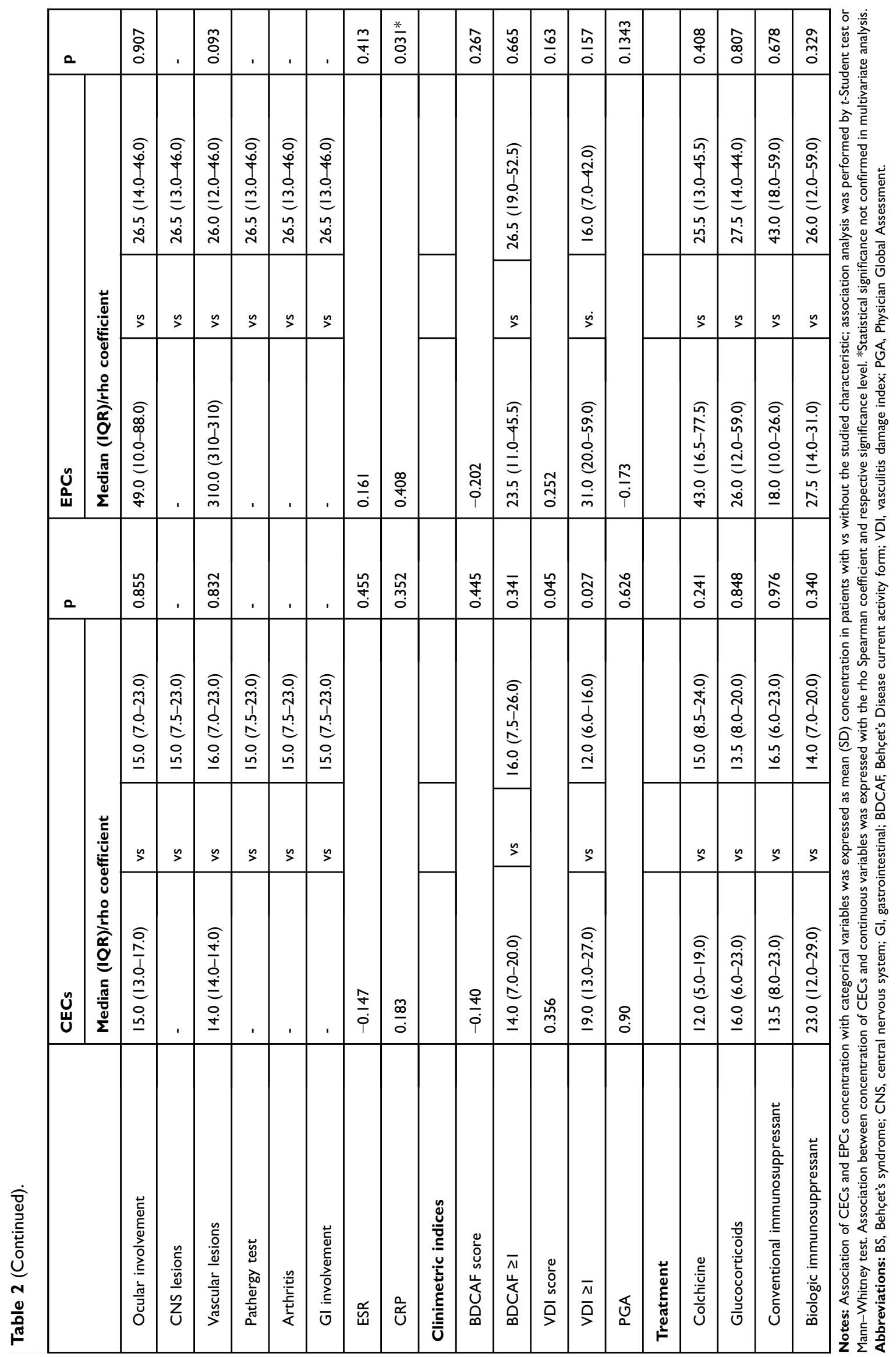




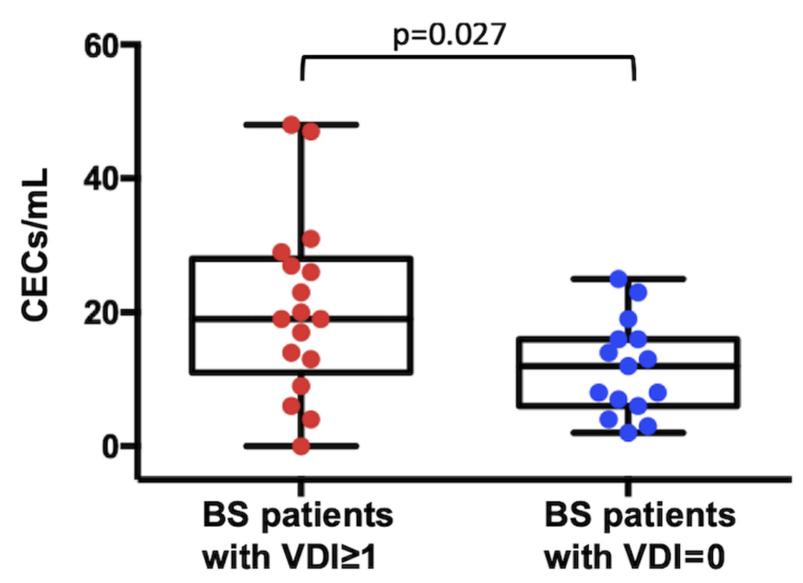

Figure 3 Concentration of circulating endothelial cells (CECs) in patients affected by Behçet's syndrome with at least one item of irreversible damage and without any damage, as assessed by the vasculitis damage index (VDI). The ends of the box are the Ist and 3rd interquartile, the vertical line inside the box is the median, the whiskers represent the highest and lowest values.

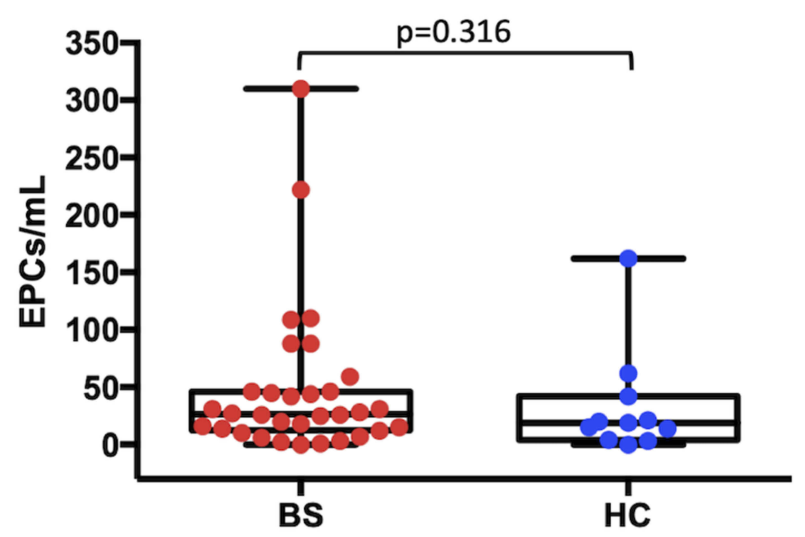

Figure 4 Concentration of endothelial progenitor cells (CECs) in Behçet's syndrome (BS) patients and healthy controls $(\mathrm{HC})$. The ends of the box are the Ist and $3 r d$ interquartile, the vertical line inside the box is the median, the whiskers represent the highest and lowest values.

endothelial cells. EPCs are currently more commonly identified by flow cytometry as CD34-positive, VEGFR2 (or CD309) positive, and CD133-positive cells, ${ }^{23}$ though many studies, including the present, typically employ two of the three receptors, mainly due to the controversial utility of CD133. ${ }^{24-26}$

The main strength of the present study is that it is the first work specifically focused on BS which assessed both CECs and EPCs concentration by using a standardized flow cytometry protocol, providing highly reliable data especially for CECs.

On the other hand, the main limits of the study were the small size of the cohort, especially in the control group, and the relatively low number of patients with high disease activity. These factors might have prevented to detect significant differences in concentration of EPCs in BS patient and HC, as well as significant associations of CECs and EPCs with disease activity. Further, at present, the lack of a longitudinal assessment did not allow to evaluate the potential correlation between modifications in the concentration of these cells and treatment or changes in disease activity. However, in the context of a complete lack of valid biomarkers for BS, these explorative data provide a relevant contribute in understanding the potential role of these cells in BS and in planning future research in the field.

In conclusion, our study suggests that the CECs concentration is significantly higher in BS than healthy subjects, and it correlates with vascular damage, even not currently active. A longitudinal extension of the present study on a wider cohort would be useful to validate the potential role of CECs as a marker or, hopefully, predictor of vascular damage in BS.

\section{Abbreviations}

CECs, circulating endothelial cells; EPCs, endothelial progenitor cells; BS, Behçet syndrome; HC, healthy controls; $\mathrm{AAV}$, anti-neutrophil cytoplasmic antibody-associated vasculitis; ICBD, International Criteria for Behçet's Disease; ESR, erythrocyte sedimentation rate; CRP, C-reactive protein; BDCAF, Behçet's Disease Current Activity Form; PGA, Physician Global Assessment; OMERACT, Outcome Measures in Rheumatology; VDI, vasculitis damage index.

\section{Ethics Statement}

The study was approved by the Local Ethical Committee of the Azienda Ospedaliero Universitaria di Cagliari (PG/ 2018/6028), and written informed consent was obtained from all subjects. All procedures were in accordance with the Good Clinical Practice standards and Helsinki Declaration.

\section{Acknowledgments}

The authors thank all recruited patients and control subjects for their participation in this study. We thank the Associazione Italiana Malattia Sindrome di Behçet (SIMBA) for partially funding this study and the Italian Society of Rheumatology (Società Italiana di Reumatologia - SIR) for supporting by a bursary to the author Alberto Floris. 


\section{Disclosure}

The authors report no conflicts of interest in this work.

\section{References}

1. Sakane T, Takeno M, Suzuki N, Inaba G. Behçet's disease. $N$ Engl $J$ Med. 1999;341(17):1284-1291. doi:10.1056/NEJM199910213411707

2. Marshall SE. Behçet's disease. Best Pract Res Clin Rheumatol. 2004;18(3):291-311. doi:10.1016/j.berh.2004.02.008

3. McGonagle D, Aydin SZ, Gül A, Mahr A, Direskeneli H. 'MHC-Iopathy'-unified concept for spondyloarthritis and Behçet disease. Nat Rev Rheumatol. 2015;11(12):731-740. doi:10.1038/nrrheum.2015.147

4. Piga M, Mathieu A. The origin of Behçet's disease geoepidemiology: possible role of a dual microbial-driven genetic selection. Clin Exp Rheumatol. 2014;32(4 Suppl 84):S123-S129.

5. Piga M, Mathieu A. Genetic susceptibility to Behcet's disease: role of genes belonging to the MHC region. Rheumatol Oxf Engl. 2011;50 (2):299-310. doi:10.1093/rheumatology/keq331

6. Angioni MM, Piga M, Paladini F, et al. AIF-1 gene does not confer susceptibility to Behçet's disease: analysis of extended haplotypes in Sardinian population. PLoS One. 2018;13(9):e0204250. doi:10.1371/ journal.pone. 0204250

7. Gündüz Ö. Histopathological evaluation of Behçet's disease and identification of new skin lesions. Patholog Res Int. 2012;2012:209316.

8. Kobayashi M, Ito M, Nakagawa A, et al. Neutrophil and endothelial cell activation in the vasa vasorum in vasculo-Behçet disease. Histopathology. 2000;36(4):362-371. doi:10.1046/j.1365-2559.2000.00859.x

9. Lanuti P, Rotta G, Almici C, et al. Endothelial progenitor cells, defined by the simultaneous surface expression of VEGFR2 and $\mathrm{CD} 133$, are not detectable in healthy peripheral and cord blood. Cytometry A. 2016;89(3):259-270. doi:10.1002/cyto.a.22730

10. Lanuti P, Simeone P, Rotta G, et al. A standardized flow cytometry network study for the assessment of circulating endothelial cell physiological ranges. Sci Rep. 2018;8(1):5823. doi:10.1038/s41598018-24234-0

11. Haubitz M, Woywodt A. Circulating endothelial cells and vasculitis. Intern Med. 2004;43(8):660-667. doi:10.2169/internalmedicine.43.660

12. Woywodt A, Blann AD, Kirsch T, et al. Isolation and enumeration of circulating endothelial cells by immunomagnetic isolation: proposal of a definition and a consensus protocol. J Thromb Haemost. 2006;4 (3):671-677. doi:10.1111/j.1538-7836.2006.01794.x

13. Nakatani K, Takeshita S, Tsujimoto H, Kawamura Y, Tokutomi T, Sekine I. Circulating endothelial cells in Kawasaki disease. Clin Exp Immunol. 2003;131(3):536-540. doi:10.1046/j.1365-2249.2003.02091.x

14. Závada J, Kideryová L, Pytlík R, Vaňková Z, Tesař V. Circulating endothelial progenitor cells in patients with ANCA-associated vasculitis. Kidney Blood Press Res. 2008;31(4):247-254. doi:10.1159/ 000142690
15. Kutlay S, Calayoglu R, Boyvat A, et al. Circulating endothelial cells: a disease activity marker in Behçet's vasculitis? Rheumatol Int. 2008;29(2):159. doi:10.1007/s00296-008-0656-y

16. Keşkek ŞÖ, Bozkırlı-Ersözlü ED, Kozanoglu I, Yücel AE. High levels of circulating endothelial progenitor cells are associated with acrotism in patients with takayasu arteritis. Med Princ Pract. 2017;26 (2):132-138. doi:10.1159/000453038

17. International Team for the Revision of the International Criteria for Behçet's Disease (ITR-ICBD), Davatchi F, Assaad-Khalil S, et al. The International Criteria for Behçet's Disease (ICBD): a collaborative study of 27 countries on the sensitivity and specificity of the new criteria. J Eur Acad Dermatol Venereol. 2014;28(3):338-347. doi:10. 1111/jdv.12107.

18. Lawton G, Bhakta BB, Chamberlain MA, Tennant A. The Behçet's disease activity index. Rheumatology. 2004;43(1):73-78. doi:10.10 93/rheumatology/keg453

19. Exley AR, Bacon PA, Luqmani RA, et al. Development and initial validation of the vasculitis damage index for the standardized clinical assessment of damage in the systemic vasculitides. Arthritis Rheum. 1997;40(2):371-380. doi:10.1002/art.1780400222

20. Exley AR, Carruthers DM, Luqmani RA, et al. Damage occurs early in systemic vasculitis and is an index of outcome. QJM. 1997;90 (6):391-399. doi:10.1093/qjmed/90.6.391

21. Suppiah R, Flossmann R, Mukhtyar C. Measurement of damage in systemic vasculitis: a comparison of the Vasculitis Damage Index with the Combined Damage Assessment Index. Ann Rheum Dis. 2011;70(1):80-85. doi:10.1136/ard.2010.139832

22. Bozkirli EDE, Keşkek SÖ, Kozanoğlu I, Yücel AE. High levels of endothelial progenitor cells can be associated with thrombosis in patients with Behçet's disease. Clin Exp Rheumatol. 2014;32(4 Suppl 84):S49-S53.

23. Del Papa N, Pignataro F. The role of endothelial progenitors in the repair of vascular damage in systemic sclerosis. Front Immunol. 2018;9. doi:10.3389/fimmu.2018.01383

24. Simard T, Jung RG, Motazedian P, et al. Progenitor cells for arterial repair: incremental advancements towards therapeutic reality. Stem Cells Int. 2017;2017:8270498. doi:10.1155/2017/8270498

25. Werner N, Kosiol S, Schiegl T, et al. Circulating endothelial progenitor cells and cardiovascular outcomes. $N$ Engl J Med. 2005;353 (10):999-1007. doi:10.1056/NEJMoa043814

26. Sirker AA, Astroulakis ZMJ, Hill JM. Vascular progenitor cells and translational research: the role of endothelial and smooth muscle progenitor cells in endogenous arterial remodelling in the adult. Clin Sci. 2009;116(4):283-299. doi:10.1042/CS20080001
Open Access Rheumatology: Research and Reviews

\section{Publish your work in this journal}

Open Access Rheumatology Research and Reviews is an international, peer-reviewed, open access journal publishing original research, reports, editorials, reviews and commentaries on all aspects of clinical and experimental rheumatology in the clinic and laboratory including the following topics: Pathology, pathophysiology of rheumatological diseases; Investigation, treatment and management of rheumatological diseases; Clinical trials and novel pharmacological approaches for the treatment of rheumatological disorders. The manuscript management system is completely online and includes a very quick and fair peer-review system, which is all easy to use. Visit http://www.dovepress.com/testimonials.php to read real quotes from published authors. 